\title{
Faire de la physique autour des fontaines lasers
}

Sébastien Forget, Christophe Daussy et Paul-Éric Pottie (pottie@univ-paris13.fr)

Laboratoire de Physique des Lasers, UMR 7538 CNRS, Université Paris 13, 99 av. J.-B. Clément, 93430 Villetaneuse

Les fontaines lumineuses

ont connu ces dernières

années en France un nouvel

essor. Grâce à la directivité

et à l'intensité des faisceaux

lasers, les fontaines lasers

permettent d'expérimenter

de façon ludique et specta-

culaire les bases de l'optique

géométrique et la vision

des couleurs.

Nous décrivons les principaux

enjeux pédagogiques

de l'expérience, ainsi que

les différentes fontaines

développées au Laboratoire

de Physique des Lasers (LPL).

Cela va de la plus simple et

la plus économique, destinée

à être reproduite aisément,

jusqu'à la Fontaine laser

géante, réalisée dans le cadre d'un projet « art et science »

pour la célébration des 50 ans du laser en 2010.
Au milieu du xix ${ }^{\mathrm{e}}$ siècle, Jean-Daniel Colladon eut l'idée de concentrer les rayons issus d'une lampe sur l'embouchure du jet d'eau créé par un réservoir percé sur un de ses flancs. Le résultat, qu'il décrit lui-même, est spectaculaire : "la lumière circule dans ce jet transparent, comme dans un canal, et en suit toutes les inflexions!» [1]. Une expérience similaire fut réalisée dès 1840 par Jacques Babinet [2] : ce dernier expliqua même comment le principe du guidage de la lumière pouvait s'étendre à des cylindres de verre courbés, anticipant ainsi les futures fibres optiques... Les fontaines lumineuses, initialement conçues dans un but pédagogique, ont rapidement connu un succès remarquable (dans des versions plus imposantes) lors de diverses manifestations en Europe et aux USA, dont l'Exposition universelle de Paris en 1889.

Grâce au laser, dont on célèbre cette année le jubilé, les fontaines lumineuses ont fait l'objet d'un regain d'intérêt en devenant des fontaines lasers (fig. 1). En exploitant les propriétés spécifiques de cette lumière (monochromaticité et faible divergence du faisceau), les fontaines lasers permettent d'expérimenter de façon simple et visuelle les bases de l'optique géométrique et la vision des couleurs. Ces notions intéressent un très large public : les plus jeunes (classes élémentaires ou collège) pour le mélange des couleurs dans l'eau par synthèse additive, les lycéens et étudiants pour expliquer le laser et le guidage de la lumière par réflexion totale. Plusieurs fontaines lasers ont été développées en France pour des actions de communication et de vulgarisation scientifique: au laboratoire PIIM (Marseille), au laboratoire ENSSAT (Lannion), au LAAS (Toulouse), dans le Scientibus (Limoges)... Au Laboratoire de physique des lasers (LPL) de l'Université Paris 13, trois fontaines ont été développées depuis 2007, et présentées lors de multiples manifestations scientifiques.

〉〉

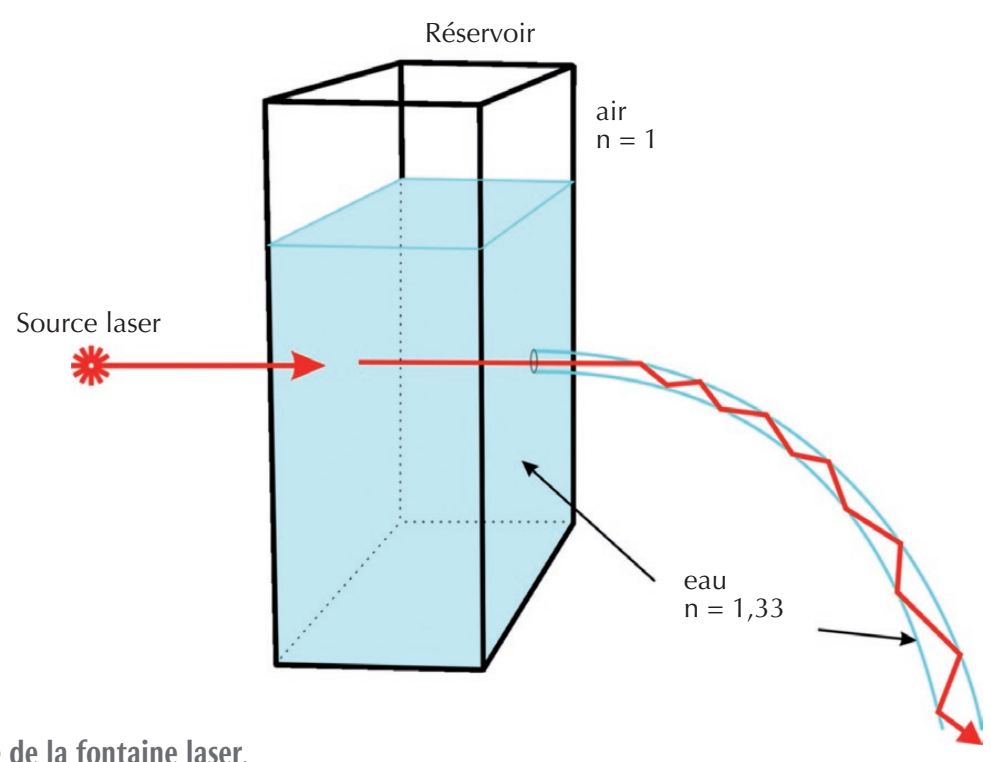

1. Principe de la fontaine laser. 


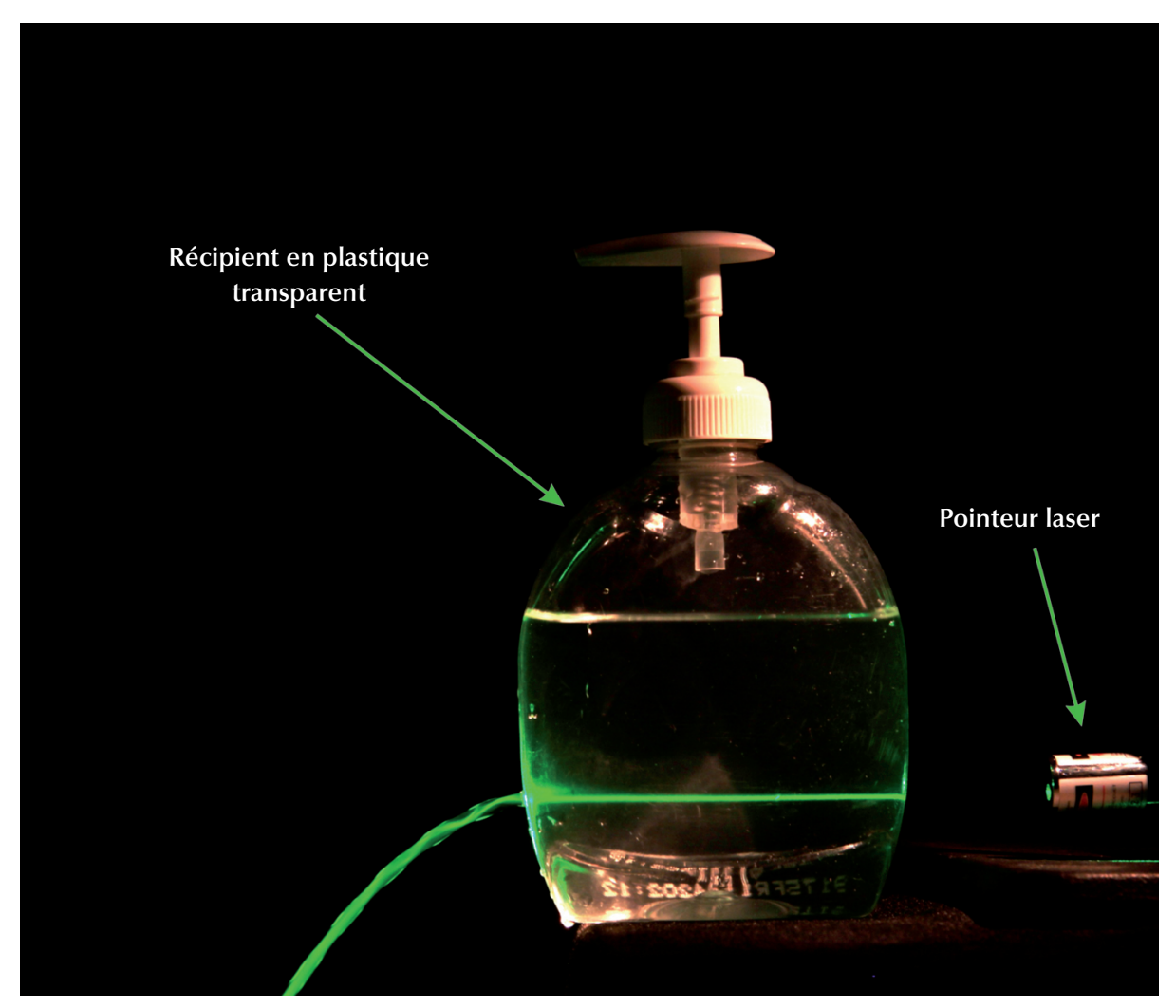

2. Fontaine à 10 euros.

〉〉

\section{Les objectifs pédagogiques}

Il s'agit tout d'abord d'expliquer le guidage de la lumière par réflexion totale dans les jets d'eau, ces derniers formant une version macroscopique des fibres optiques. Ici, le milieu guidant est aqueux et non vitreux, et la "gaine » est faite d'air : les notions fondamentales (contraste d'indice avec un indice plus fort dans le cœur que dans la gaine, réflexion totale, courbure possible) sont bien respectées. Dans le cas du guidage dans un jet d'eau, la différence entre l'indice du cœur $\left(\mathrm{n}_{\text {eau }}=1,33\right)$ et celui de la gaine $\left(\mathrm{n}_{\text {air }}=1\right)$ est bien plus importante que pour une fibre optique classique (où elle vaut quelques millièmes), et l'injection s'en trouve grandement facilitée. Un point délicat de ces dispositifs est de créer un jet d'eau suffisamment peu turbulent (nombre de Reynolds inférieur à 3000). Le calcul de la vitesse de l'écoulement repose sur l'application de l'équation de Bernoulli, et le calcul de la trajectoire du jet d'eau reste simple: quelques notions de base de mécanique des fluides peuvent ainsi être introduites. L'utilisation d'un laser, au lieu d'une simple lampe, permet de profiter de son faisceau très directif pour visualiser distinctement la première réflexion totale dans le jet d'eau, les suivantes étant souvent brouillées par la turbulence du jet.
Après une ou deux réflexions totales, le jet d'eau apparaît uniformément coloré, ce qui permet une observation directe de la synthèse additive des couleurs en injectant dans un même jet des faisceaux lasers rouge, vert et bleu (les trois couleurs primaires). En modifiant l'intensité respective de ces lasers, on peut alors faire varier la couleur du jet d'eau et obtenir toutes les couleurs (y compris le blanc). Un effet esthétique intéressant peut être obtenu par une variation aléatoire de l'intensité des trois lasers (via une commande électronique), créant un changement continuel de la couleur du jet.

Enfin, de multiples ouvertures peuvent être proposées autour de cette expérience : le laser et ses nombreuses applications (dans l'industrie, la médecine ou la recherche), les télécommunications optiques, ou encore les applications quotidiennes de la synthèse additive (écrans) et soustractive (imprimantes).

\section{Aspects techniques et réalisations pratiques}

\section{Une fontaine laser à 10 euros}

Une première expérience, peu onéreuse et accessible à tous (en classe ou à la maison), permet d'observer très simplement la réflexion totale. L'expérience est réalisée avec un simple pointeur laser rouge ou vert (puissance $<1 \mathrm{~mW}$, classe 2 ) et un récipient en plastique transparent avec des faces parallèles, l'une d'elles percée d'un trou. Le récipient est rempli d'eau, puis le faisceau lumineux est dirigé vers le trou afin d'observer la lumière guidée dans le jet (fig. 2).

\section{Une fontaine à trois lasers pour mélanger les couleurs}

Nous avons développé une fontaine laser plus élaborée, destinée aux actions de diffusion de la connaissance et de vulgarisation scientifique du LPL [3]. Ce montage permet d'observer, en plus de la réflexion totale, la synthèse additive des couleurs à partir des trois couleurs primaires (fig. 3).

Des lasers de quelques dizaines de milliwatts (classe 3B) et un bon contraste sont nécessaires pour obtenir un effet saisissant. Trois faisceaux lasers, rouge, vert et bleu (respectivement une diode de $50 \mathrm{~mW}$ à $650 \mathrm{~nm}$, deux Nd:YAG doublés en fréquence de $50 \mathrm{~mW}$ à $532 \mathrm{~nm}$ et de $20 \mathrm{~mW}$ à $473 \mathrm{~nm}$ ), sont injectés et mélangés dans plusieurs jets d'eau à l'aide d'éléments optiques (miroirs, lames séparatrices, cubes et/ou prismes). L'intensité des lasers peut être modifiée par un simple jeu de densités, 


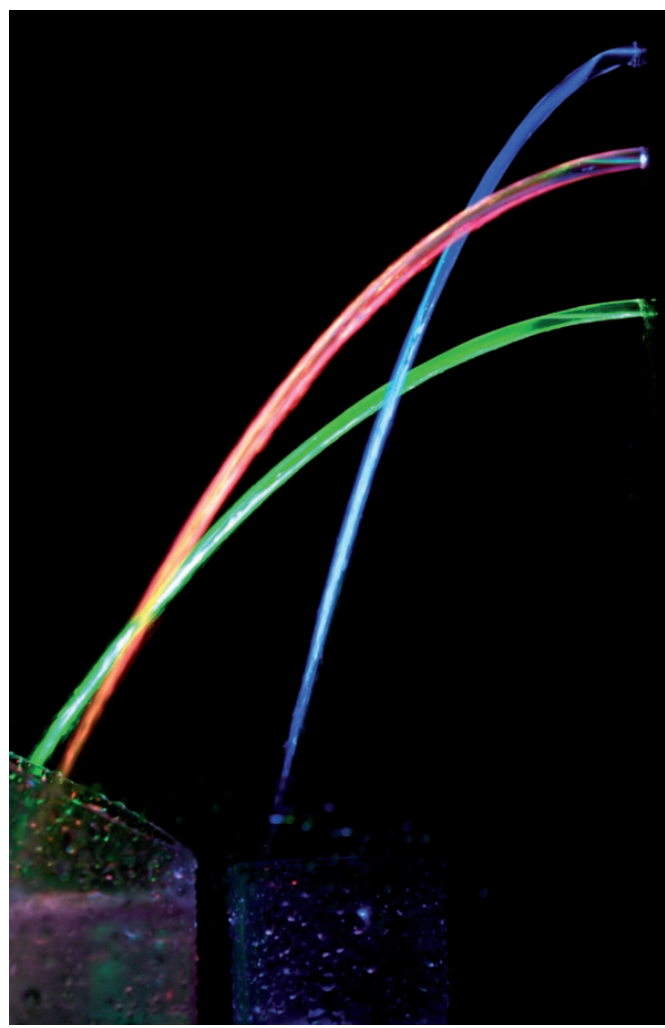

3. Mélange des couleurs dans la fontaine à trois lasers.

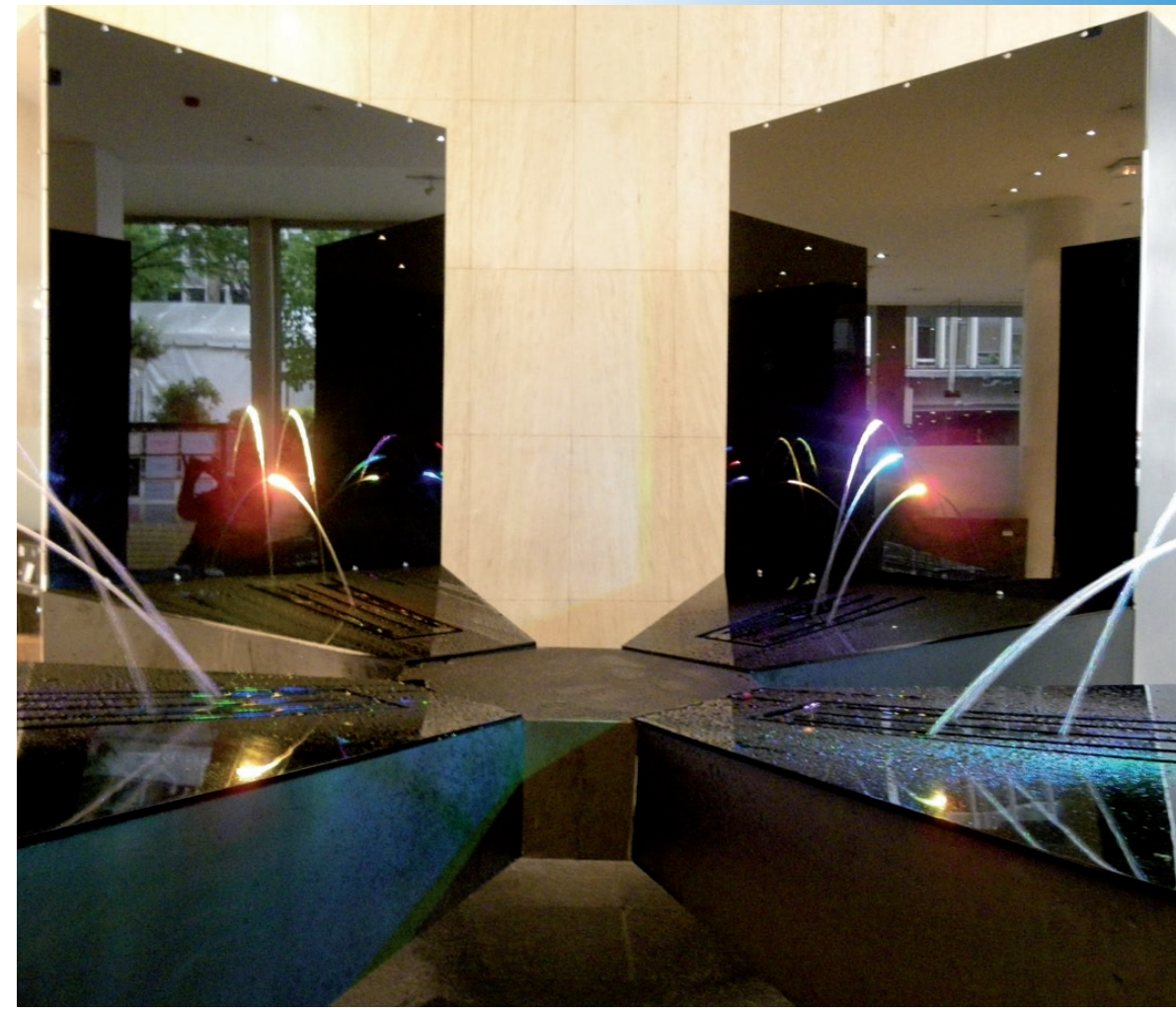

4. La Fontaine laser géante en avant-première, au siège du CNRS, le 1er juin 2010. ou bien en ajustant le rapport cyclique ${ }^{(1)}$ d'un signal logique appliqué à chaque laser, à l'aide d'un microcontrôleur. Pour assurer un fonctionnement en continu, l'eau des jets est récupérée dans un bassin, puis recyclée jusqu'au réservoir par une petite pompe. Une portée des jets de 10 à $30 \mathrm{~cm}$ est obtenue avec une hauteur de colonne d'eau de 10 à $20 \mathrm{~cm}$ et des trous d'environ $6 \mathrm{~mm}$ de diamètre. Le débit ajustable de la pompe permet de faire varier la portée et la turbulence des jets. La fontaine est placée dans une grande boîte noire $\left(1,2 \times 0,6 \times 0,7 \mathrm{~m}^{3}\right)$, afin d'assurer une observation de jets d'eau lumineux sur fond noir et le confinement des rayonnements, nécessaire pour garantir la sécurité laser.

Une ouverture vers 1'art :

la Fontaine laser géante

À l'occasion de la célébration des 50 ans du laser, en 2010, le LPL a été porteur d'un projet " art et science " pour la réalisation d'une Fontaine laser géante [4-5]. Un jeune artiste, Steve Veloso [6], a relevé ce défi et a réalisé une installation jouant sur l'abstraction formelle et sur la visibilité du mécanisme interne. L'œuvre recherche un impact "physique " sur le spectateur, pour l'interroger sur son rapport à l'art et à la science. Cette fontaine, de $16 \mathrm{~m}^{2}$ au sol et de 2,6 $\mathrm{m}$ de hauteur, est composée de quatre larges monolithes noirs de métal et de plexiglas, tantôt miroitants, tantôt transparents, et d'un bassin intérieur (fig. 4). Des lasers encore plus puissants (200 mW, classe 4) et des bancs optiques plus élaborés sont installés dans le cœur des monolithes.

\section{Conclusion}

Les fontaines lasers sont d'excellents outils pour faire de la physique à tous les niveaux : celles du LPL sont régulièrement présentées lors de manifestations de communication et de vulgarisation scientifique à destination des collèges et lycées (ateliers science, Bars des Sciences, visites de scolaires...), et à l'occasion d'événements nationaux qui rassemblent plusieurs milliers de visiteurs (Nuit des Chercheurs, Fête de la Science/Savante Banlieue...).

La Fontaine laser géante a été présentée en avant-première au siège du CNRS, puis inaugurée à l'Université de Paris 13 (Villetaneuse) au mois de juin 2010. Elle sera présentée en France tout au long des années 2010 et 2011 dans divers lieux (musées, universités, CCSTI...), et participera à la cérémonie de clôture des 50 ans du laser à Bordeaux, le 2 décembre 2010 .
Remerciements

Nous tenons à remercier l'ensemble des membres du LPL qui ont participé avec enthousiasme aux nombreuses présentations des fontaines lasers et plus particulièrement A. Kaladjian, F. Wiotte, M. Fosse, B. Darquié et J.-C. Keller, pour leurs contributions décisives à ces expériences. La fontaine à trois lasers a été réalisée avec le soutien de l'Université Paris 13 et du Conseil général de SeineSaint-Denis. La Fontaine laser géante a été financée par le CNRS, l'Université Paris 13 et le LPL, avec le soutien de la Mairie de Paris, l'Institut d'Optique Graduate School, la Société Française de Physique (SFP), Sciences Essonne, ALPhA-Route des Lasers, l'Institut Galilée et le Comité national des 50 ans du laser.

(1) Le rapport cyclique est le rapport de la durée de l'état haut à la période du signal logique (TTL).

\section{Références}

1 - D. Colladon, "On the reflections of a ray of light inside a parabolic liquid stream", Comptes Rendus $\mathbf{1 5}$ (1842) 800.

2 -J. Hecht, City of Light, The Story of Fiber Optics, Oxford University Press, New York, 1999 (ISBN 0-19-510818-3).

- Site web des fontaines au LPL : www-Ipl.univ-paris13.fr:8090/fontaine_laser.htm

4 - Sites web des 50 ans du laser: www.50ansdulaser.fr www.cnrs.fr/50anslaser/spip.php?article70

5 - Site web de La Fontaine laser géante www.fontainelaser.fr

6 - Site web de Steve Veloso steveveloso.com/index.html 